\title{
Axial vector form factor of nucleons in a light-cone diquark model
}

\author{
Bo-Qiang Ma, ${ }^{1, \text { f Di Qing, }}{ }^{2, \text { I }}$ and Iván Schmidt ${ }^{2}$ 用 \\ ${ }^{1}$ Department of Physics, Peking University, Beijing 100871, China \\ ${ }^{2}$ Departamento de Física, Universidad Técnica \\ Federico Santa María, Casilla 110-V, Valparaíso, Chile
}

\begin{abstract}
The nucleon axial vector form factor is investigated in a light-cone quark spectator diquark model, in which Melosh rotations are applied to both the quark and vector diquark. It is found that this model gives a very good description of available experimental data and the results have very little dependence on the parameters of the model. The relation between the nucleon axial constant and the anomalous magnetic moment of nucleons is also discussed.
\end{abstract}

PACS numbers: 13.10.+q, 12.39.Ki, 14.20.Dh

*Electronic address: mabq@phy.pku.edu.cn

$\dagger$ Electronic address: diqing@fis.utfsm.c]; corresponding author.

‡Electronic address: ischmidt@fis.utfsm.c 
Since the momenta of quarks within a hadron are of the same order as the masses of quarks, fully relativistic quark models are required in order to describe hadron transition processes. The light-cone quantization provides such a fully relativistic treatment with lots of unique properties [1]. It is well known that the matrix elements of local operators such as the electromagnetic and weak currents have exact representation in terms of light-cone wave functions of Fock states [2]. If one chooses the special frame [3] $q^{+}=0$ for the spacelike momentum transfer and takes the matrix elements of plus components of currents, the contribution from pair creation or annihilation is forbidden and the matrix elements of spacelike currents can be expressed as overlaps of light-cone wave functions with the same number of Fock constituents. Therefore, the light-cone frame is well suited for the description of electromagnetic and weak transition processes.

In previous papers, a light-cone quark spectator diquark model was proposed in order to investigate the nucleon spin problem [4, 5, 6. This model is based on the assumption that deep inelastic scattering is well described by the impulse approximation picture of the quarkparton model [7], in which the incident lepton scatters incoherently off a quark in the nucleon, with the remaining nucleon constituents treated as a quasi-particle spectators to provide the remaining nucleon quantum number. In fact, in the quark spectator diquark form, some nonperturbative effects between the two spectator quarks or other non-perturbative gluon effects in the nucleon can be effectively taken into account by the mass of the diquark spectator. After taking into account Melosh rotation effects, this model is in good agreement with experimental data of polarized deep inelastic scattering, and the mass difference between the scalar and vector spectators reproduces the up and down valence quark asymmetry [4, 5, 6.

Recently, based on the impulse approximation, this model was extended to study the electromagnetic form factors of nucleons and the results agree with experiment [8]. After applying Melosh rotations to both quark and spectator vector diquark, the difference between the scalar and vector diquarks breaks the $S U(6)$ symmetry of nucleon wave functions and reproduces the correct electromagnetic nucleon properties, especially in the case of the neutron. It is natural to extend the light-cone quark diquark model to study other transition processes, such as the nucleon axial decay transition process which is important in the study of the structure of nucleons [9]. This is clearly a non-trivial extension, since we are going now into the weak interactions domain. 
The nucleon axial vector form factor $G_{A}\left(Q^{2}\right)$ is defined by

$$
\left\langle P^{\prime}, S^{\prime}\left|A_{a}^{\mu}(0)\right| P, S\right\rangle=\bar{u}\left(P^{\prime}, S^{\prime}\right)\left[G_{A}\left(Q^{2}\right) \gamma^{\mu}+G_{P}\left(Q^{2}\right) \frac{q_{\mu}}{2 M}\right] \gamma_{5} \frac{\tau_{a}}{2} u(P, S)
$$

where $A_{a}^{\mu}(0)$ is the axial vector current, $q^{\mu}=\left(P^{\prime}-P\right)^{\mu}$ is four-momentum transfer, $Q^{2}=$ $-q^{2}, u(P, S)$ is the nucleon spinor, and $\tau_{a}$ is the isospin matrix with Cartesian index $a$. In the light-cone frame, the plus component of the axial vector current reads

$$
\frac{\bar{u}\left(k^{\prime}, \uparrow\right)}{\sqrt{k^{\prime+}}} \gamma^{+} \gamma_{5} \frac{u(k, \uparrow)}{\sqrt{k^{+}}}=-\frac{\bar{u}\left(k^{\prime}, \downarrow\right)}{\sqrt{k^{\prime+}}} \gamma^{+} \gamma_{5} \frac{u(k, \downarrow)}{\sqrt{k^{+}}}=2,
$$

and in these calculations we choose the Drell-Yan assignment [3]:

$$
\begin{aligned}
q & =\left(q^{+}, q^{-}, \vec{q}_{\perp}\right)=\left(0, \frac{-q^{2}}{P^{+}}, \vec{q}_{\perp}\right), \\
P & =\left(P^{+}, P^{-}, \vec{P}_{\perp}\right)=\left(P^{+}, \frac{M^{2}}{P^{+}}, \overrightarrow{0}_{\perp}\right),
\end{aligned}
$$

thus we have

$$
G_{A}\left(Q^{2}\right)=\left\langle P^{\prime}, \uparrow\left|\frac{A^{+}(0)}{2 P^{+}}\right| P, \uparrow\right\rangle .
$$

Thus, in similar to the electromagnetic operators, the matrix element of axial vector currents can be expressed in the light-cone formalism as overlaps of light-cone wave functions with the same number of Fock constituents as:

$$
G_{A}\left(Q^{2}\right)=\sum_{a} \int \frac{d^{2} \vec{k}_{\perp} d x}{16 \pi^{3}} \sum_{j} \tau_{j} \lambda_{j} \psi_{a}^{\uparrow \star}\left(x_{i}, \vec{k}_{\perp i}^{\prime}, \lambda_{i}\right) \psi_{a}^{\uparrow}\left(x_{i}, \vec{k}_{\perp i}, \lambda_{i}\right)
$$

where $\tau_{j}$ and $\lambda_{j}$ are the isospin and helicity of the struck constituents, $\psi_{a}^{\uparrow}\left(x_{i}, \vec{k}_{\perp i}^{\prime}, \lambda_{i}\right)$ is the light-cone Fock expansion wave function, and $\lambda_{i}, x_{i}$ and $\vec{k}_{\perp i}$ are the spin projections along the quantization $z$ direction, light-cone momentum fractions and relative momentum coordinates of QCD constituents, respectively. Here, for the final state light-cone wave function, the relative momentum coordinates are

$$
\vec{k}_{\perp i}^{\prime}=\vec{k}_{\perp i}+\left(1-x_{i}\right) \vec{q}_{\perp}
$$

for the struck quark and

$$
\vec{k}_{\perp i}^{\prime}=\vec{k}_{\perp i}-x_{i} \vec{q}_{\perp}
$$

for each spectator. 
In this work we study the nucleon axial vector form factor based on the light-cone quark spectral diquark model [B], in which the Melosh rotations is applied to both quark and diquark, explicitly

$$
\begin{aligned}
& \chi_{T}^{\uparrow}=w\left[\left(k^{+}+m\right) \chi_{F}^{\uparrow}-k^{R} \chi_{F}^{\downarrow}\right], \\
& \chi_{T}^{\downarrow}=w\left[\left(k^{+}+m\right) \chi_{F}^{\downarrow}-k^{L} \chi_{F}^{\uparrow}\right],
\end{aligned}
$$

for quarks [10], and

$$
\begin{aligned}
V_{T}^{1}= & w^{2}\left[\left(k^{+}+m\right)^{2} V_{F}^{1}-\sqrt{2}\left(k^{+}+m\right) k^{R} V_{F}^{0}+k^{R^{2}} V_{F}^{-1}\right], \\
V_{T}^{0}= & w^{2}\left[\sqrt{2}\left(k^{+}+m\right) k^{L} V_{F}^{1}+2\left(\left(k^{0}+m\right) k^{+}-k^{R} k^{L}\right) V_{F}^{0}\right. \\
& \left.-\sqrt{2}\left(k^{+}+m\right) k^{R} V_{F}^{-1}\right], \\
V_{T}^{-1}= & w^{2}\left[k^{L^{2}} V_{F}^{1}+\sqrt{2}\left(k^{+}+m\right) k^{L} V_{F}^{0}+\left(k^{+}+m\right)^{2} V_{F}^{-1}\right],
\end{aligned}
$$

for vector diquarks [11]. Here, $\chi_{T}$ and $\chi_{F}$ are instant and light-cone spin- $\frac{1}{2}$ spinors, $V_{T}$ and $V_{F}$ are the instant and light-cone spin-1 spinors respectively, $w=\left[2 k^{+}\left(k^{0}+m_{q}\right)\right]^{-\frac{1}{2}}$, $k^{R, L}=k^{1} \pm i k^{2}$, and $k^{+}=k^{0}+k^{3}$. And the details of the quark diquark model can be found in Ref. [8]. Therefore, according to Eq. (5), we have

$$
\begin{aligned}
G_{A}\left(Q^{2}\right)= & 3 \int \frac{d^{2} k_{\perp} d x}{16 \pi^{3}} w_{q}^{\prime} w_{q}\left\{\frac { 1 } { 9 } \operatorname { s i n } ^ { 2 } \theta \left\{-\left[\left(k_{q}^{\prime+}+m_{q}\right)\left(k_{q}^{+}+m_{q}\right)-k_{\perp}^{\prime L} k_{\perp}^{R}\right]\right.\right. \\
& \times O_{V^{0,0}}+\sqrt{2}\left[\left(k_{q}^{++}+m_{q}\right) k_{\perp}^{L}+\left(k_{q}^{+}+m_{q}\right) k_{\perp}^{\prime L}\right] O_{V^{0,1}} \\
& +\sqrt{2}\left[\left(k_{q}^{\prime+}+m_{q}\right) k_{\perp}^{R}+\left(k_{q}^{+}+m_{q}\right) k_{\perp}^{\prime R}\right] O_{V^{1,0}} \\
& \left.+2\left[\left(k_{q}^{\prime+}+m_{q}\right)\left(k_{q}^{+}+m_{q}\right)-k_{\perp}^{\prime R} k_{\perp}^{L}\right] O_{V^{1,1}}\right\} \varphi_{V}\left(x, \vec{k}_{\perp}^{\prime}\right) \\
& \times \varphi_{V}\left(x, \vec{k}_{\perp}\right)+\cos ^{2} \theta\left[\left(k_{q}^{\prime+}+m_{q}\right)\left(k_{q}^{+}+m_{q}\right)-k_{\perp}^{\prime L} k_{\perp}^{R}\right] \\
& \left.\times \varphi_{S}\left(x, \vec{k}_{\perp}^{\prime}\right) \varphi_{S}\left(x, \vec{k}_{\perp}\right)\right\},
\end{aligned}
$$

where $O_{V}$ come from the Melosh rotation of vector diquarks, and $\varphi$ are the momentum space wave function which is assumed to be a harmonic oscillator wave function (the BrodskyHuang-Lepage (BHL) prescription [12]).

Following Ref. [8], we calculate the nucleon axial vector form factor using three different sets of parameters in order to show its dependence on the difference between the scalar and vector diquarks. This is shown in Fig. 1. As Ref. [13], the experimental data are assuming to be the dipole form

$$
G_{A}\left(Q^{2}\right)=\frac{g_{A}}{\left(1+Q^{2} / M_{A}^{2}\right)^{2}}
$$


where the axial constant $g_{A}=1.2670(35)$ is from the most recent review by Particle Data Group [14], and $M_{A}$ is the axial mass. Our results agree with the experiment very well. In contrast to the case of nucleon electromagnetic form factors, the nucleon axial vector form factor is largely parameter independent. Alternatively, the effect of the difference between the scalar and vector diquarks to the the nucleon axial vector form factor is small. The static properties corresponding to the axial vector current, axial constant $g_{A}$ and axial

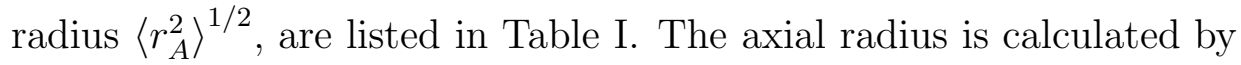

$$
r_{A}^{2}=-\left.6 \frac{1}{G_{A}(0)} \frac{d G_{A}\left(Q^{2}\right)}{d Q^{2}}\right|_{Q^{2}=0} .
$$

Our results are very close to the experimental data, and also the effect of the difference between the scalar and vector diquarks to the the nucleon axial vector static properties is small.

For the axial constant $g_{A}=G_{A}(0)$, the relativistic effects are essential in order to reduce the $S U(6)$ nonrelativistic result $g_{A}^{N R}=5 / 3$ to the experimental data. In fact, from Eq. (10) we have

$$
g_{A}=\int \frac{d^{2} k_{\perp} d x}{16 \pi^{3}}\left[\frac{1}{6} W_{A}\left(x, \vec{k}_{\perp}\right) \varphi_{V}^{2}\left(x, \vec{k}_{\perp}\right)+\frac{3}{2} W_{A}\left(x, \vec{k}_{\perp}\right) \varphi_{S}^{2}\left(x, \vec{k}_{\perp}\right)\right],
$$

where

$$
W_{A}\left(x, \vec{k}_{\perp}\right)=\frac{\left(k_{q}^{+}+m_{q}\right)^{2}-\vec{k}_{\perp}^{2}}{\left(k_{q}^{+}+m_{q}\right)^{2}+\vec{k}_{\perp}^{2}}
$$

is the Wigner rotation factor corresponding to the contribution from the relativistic effects due to the quark transversal motion 4, 5, 15. In the case of $\mathrm{SU}(6)$ symmetry between the vector and scalar diquarks, we get the same results as other light-cone quark models [15, [16], $g_{A}=\left\langle W_{A}\right\rangle g_{A}^{N R}$. In the nonrelativistic limit, $\left\langle W_{A}\right\rangle=1$ and $g_{A}=g_{A}^{N R}$. Therefore, the physical value of the axial constant is reduced $25 \%$ from its nonrelativistic value due to relativistic effects. This is similar to the nucleon spin problem situation [4], where relativistic effects are important for producing the quark spin reduction on the light-cone. This is reasonable, since from Eq. (5) it is easy to obtain the following relation between the nucleon axial constant and the quark spin contributions to the nucleon spin,

$$
g_{A}=\Delta u-\Delta d
$$

Here $\Delta u$ and $\Delta d$ are the helicity of up and down quarks in the nucleon, and $\Delta q=$ $\left\langle W_{A}\right\rangle \Delta q^{N R}$, where $\Delta q^{N R}$ is the nonrelativistic quark spin contributions to the nucleon spin defined in the quark model. 
On the other side, in our model the anomalous magnetic moment of proton $a$ can be also written as [8]

$$
\begin{aligned}
a & =2 M \int \frac{d^{2} k_{\perp} d x}{16 \pi^{3}} \frac{1}{\mathcal{M}}\left[\frac{(1-x) \mathcal{M}\left(k_{q}^{+}+m_{q}\right)-\vec{k}_{\perp}^{2} / 2}{\left(k_{q}^{+}+m_{q}\right)+\vec{k}_{\perp}^{2}}\right] \varphi_{S}^{2}\left(x, \vec{k}_{\perp}\right) \\
& =\left\langle W_{M}\right\rangle a^{N R},
\end{aligned}
$$

where $a^{N R}=2 M / 3 m_{q}$ is the nonrelativistic value of the proton anomalous magnetic moment, and the relativistic effect corresponding to the anomalous magnetic moment

$$
W_{M}=\frac{3 m_{q}}{\mathcal{M}}\left[\frac{(1-x) \mathcal{M}\left(k_{q}^{+}+m_{q}\right)-\vec{k}_{\perp}^{2} / 2}{\left(k_{q}^{+}+m_{q}\right)+\vec{k}_{\perp}^{2}}\right],
$$

which is the same as Ref. [16]. Thus, the wavefunction-independent relations between the nucleon axial-coupling $g_{A}$ and the nucleon magnetic moments of Ref. [16] are still kept in our model.

In conclusion, we extended our studies of nucleon elastic and inelastic scattering processes in a light-cone quark diquark model to the nucleon axial vector form factor and the Melosh rotations were applied to both the quark and vector diquark. It is shown that the axial vector form factor has very little dependence on the parameters of the model and the relativistic properties of the model are essential for the good agreement with the experimental results. The relation between the nucleon axial constant and the anomalous magnetic moment of nucleons is also discussed.

Therefore the light-cone quark diquark model gives a very good description of the nucleon. In fact, we have shown that it can reproduce quite accurately both elastic and inelastic electromagnetic and weak nucleon data, as can be seen by our results for the nucleon structure functions and vector and axial vector form factors.

\section{Acknowledgments}

This work is partially supported by National Natural Science Foundation of China under Grant Numbers 19975052, 10025523, and 90103007, by Fondecyt (Chile) under project 3000055 and Grant Number 8000017.

[1] S. J. Brodsky, H. C. Pauli, and S. S. Pinsky, Phys. Rep. 301, 299 (1998). 
[2] S. J. Brodsky, D. S. Huang, B.-Q. Ma, and I. Schmidt, Nucl. Phys. B593, 311 (2001).

[3] S. D. Drell and T. M. Yan, Phys. Rev. Lett. 24, 181 (1970).

[4] B.-Q. Ma, J. Phys. G 17, L53 (1991); B.-Q. Ma and Q.-R. Zhang, Z. Phys. C 58, 479 (1993).

[5] B.-Q. Ma, Phys. Lett. B 375, 320 (1996).

[6] B.-Q. Ma, I. Schmidt, and J. Soffer, Phys. Lett. B 441, 461 (1998).

[7] R. P. Feynman, Phys. Rev. Lett. 23, 1415 (1969); J. D. Bjorken, Phys. Rev. 179, 1547 (1969);

J. D. Bjorken and E. A. Paschos, Phys. Rev. 185, 1975 (1969); R. P. Feynman, in Reading, edited by Benjamin (Mass., 1972); F. E. Close, Phys. Lett. B 43, 422 (1973); R. D. Field and R. P. Feynman, Phys. Rev. D 15, 2590 (1977).

[8] B.-Q. Ma, D. Qing, and I. Schmidt, Phys. Rev. C 65, 035205 (2002).

[9] For a recent review, see V. Bernard, L. Elouadrhiri, and U.-G. Meißner, J. Phys. G 28, R1 (2002).

[10] H. J. Melosh, Phys. Rev. D 9, 1095 (1974).

[11] D. V. Ahluwalia and M. Sawicki, Phys. Rev. D 47, 5161 (1993).

[12] S. J. Brodsky, T. Huang, and G. P. Lepage, in Particles and Fields-2, edited by A. Z. Capri and A. N. Kamal (Plenum, New York, 1983), p. 143, proceedings of the Banff Summer Institute, Banff, Alberta, 1981; T. Huang, B.-Q. Ma, and Q.-X.Shen, Phys. Rev. D 49, 1490 (1994).

[13] L. Y. Glozman, M. Radici, R. F. Wagenbrunn, S. Boffi, W. Klink, and W. Plessas, Phys. Lett. B 516, 183 (2001).

[14] D. E. Groom et al., Eur. Phys. Jour. C 15, 1 (2000), particle data group, URL http://pdg. lbl.gov.

[15] P. L. Chung and F. Coester, Phys. Rev. D 44, 229 (1991).

[16] S. J. Brodsky and F. Schlumpf, Phys. Lett. B 329, 111 (1994).

[17] A. Liesenfeld et al., Phys. Lett. B 468, 20 (1999).

[18] T. Kitagaki et al., Phys. Rev. D 28, 436 (1983). 
TABLE I: The static electromagnetic properties of nucleons for the three sets of parameters.

\begin{tabular}{lcccr}
\hline \hline & Set I & Set II & Set III & Expt. \\
\hline$g_{A}$ & 1.253 & 1.270 & 1.242 & $1.2670(35)[14]$ \\
$\left\langle r_{A}^{2}\right\rangle^{1 / 2}$ & 0.624 & 0.703 & 0.611 & $0.635(23)[17], 0.65(7)[18$ \\
\hline \hline
\end{tabular}

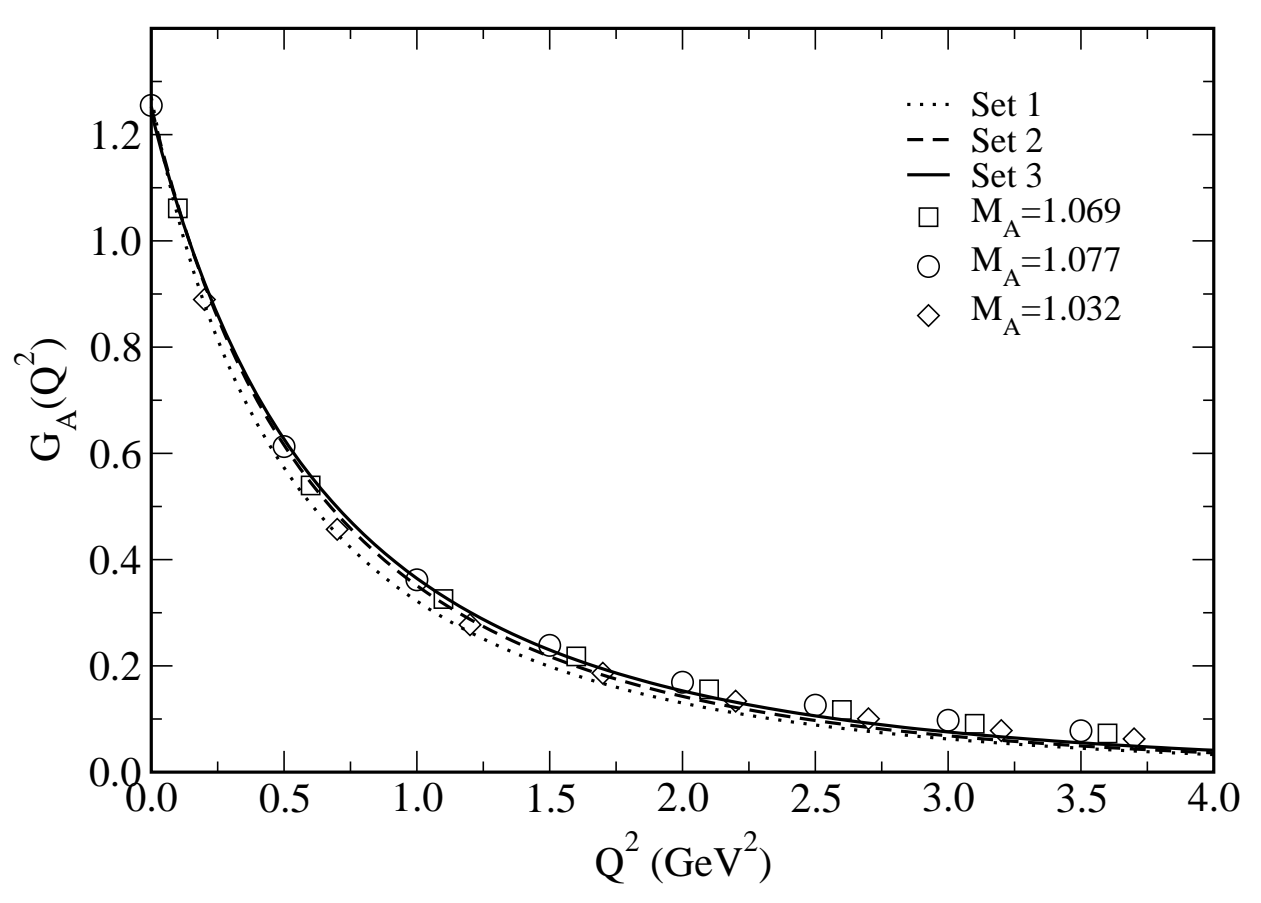

FIG. 1: Axial form factor of nucleons. The experimental data are from 17, 18 